\title{
A COMPUTATIONAL METHOD FOR THE BOUNDARY VECTOR OF A BMAP/G/1 QUEUE
}

\author{
Shoichi Nishimura \\ Tokyo University of Science
}

\author{
Takemi Shigeta \\ Tokyo University of Science, Yamaguchi
}

(Received January 14, 2005; Revised October 31, 2005)

\begin{abstract}
For calculations of the boundary vector arising in a BMAP/G/1 queue, we consider a spectral method based on eigenvalues and eigenvectors without assuming a structure of a BMAP. We define a nonlinear function of the determinant of a matrix function. It is proved that there are $M$ zeros of the nonlinear function on a disk in the complex plane, where $M$ is the size of rate matrices of a BMAP. And for the calculation of all the zeros, we propose a modification of the Durand-Kerner method which is known as an iterative method for calculating all zeros of a polynomial simultaneously. Spectral methods for calculating the stationary probabilities just after service completion epochs and at arbitrary time are given.
\end{abstract}

Keywords: Queue, BMAP/G/1, spectral method, modified Durand-Kerner method, queue length, batch arrival

\section{Introduction}

A batch Markovian arrival process (BMAP) introduced by Neuts ([10] and [11]) is great flexible and its queueing models are analytically tractable. For a BMAP/G/1 queue, several methods for calculating the stationary probability of the queue length have been studied. Matrix analytic methods in Lucantoni and Ramaswami ([7], [8], [19] and [20]) are customary techniques for analysis of a stationary probability of a BMAP/G/1 queue. Bini and Meini ([2], [3] and [9]) propose efficient methods named the cyclic reduction algorithms based on the fast Fourier transform (FFT).

All these methods are divided into two computational steps. The first is to calculate the boundary vector whose components are probabilities at idle states. The boundary vector is obtained from the stationary probability vector $\boldsymbol{g}$ of the stochastic matrix $G$ which is the minimal nonnegative solution of a matrix nonlinear equation. The second is to calculate the stationary probability from the boundary vector. Since the precision of the stationary probability strongly depends on that of the boundary vector, it is important to get the precise values of the boundary vector. It is proved in Gail et al. ([4] and [5]) that there are $M$ zeros of the determinant of a matrix function on the unit disk, where $M$ is the size of a matrix of a BMAP. And $G$ is obtained from all the zeros $z_{i}(i=1, \ldots, M)$ and the corresponding null vectors. Owing to the difficulty to find all the zeros on the unit disk, there are few implementations of this spectral method based on eigenvalues and eigenvectors.

If an underlying process of a BMAP is modulated as a birth-death process (BDMMAP), all the zeros are real and simple (Nishimura [14]). If a BMAP has a tree structure (TSMAP), it is also proved in Nishimura [13] that under regularity conditions, all the zeros are real and simple. For the first step calculation, zeros of these queueing models are calculated by the bisection method and the boundary vector is efficiently calculated. The inverse discrete 
FFT for the probability generating function discussed in [23] is applied to the second step calculation. Under the assumption of the simplicity of eigenvalues, the vector probability generating function is computed using the diagonal representation of matrixes. And the probability function of the queue length is calculated by the inverse discrete FFT for the vector probability generating function.

The objective of this paper is to represent a spectral method for the calculation of the vector $\boldsymbol{g}$ without assuming a special structure of a BMAP. It is proved in Theorem 4.3 that there are $M$ zeros of the determinant of a matrix function on a disk. And it is proved in Theorems 4.4-4.5 that the vector $\boldsymbol{g}$ is calculated by all the $M$ zeros on the disk and corresponding null vectors. Remaining our problem is how to calculate all the $M$ zeros. For this calculation we propose a modification of the Durand-Kerner method (D-K method) known as an iterative method for calculation of all zeros of a polynomial simultaneously ([1], [6], [22] and [25]).

Let $A_{k}$ be the matrix whose $(i, j)$ element is the phase transition probability from $i$ to $j$ with $k$ arrivals during the service time. In order to derive $A_{k}$, a lot of calculations and a large memory are required if a BMAP has a large batch size. Under the spectral methods, the stationary probability is derived without calculations of $A_{k}(k=0,1, \ldots)$. This is a great advantage to compute the stationary probability when we analyze a BMAP with a large batch size as IP traffic. Modeling IP traffic of the Bellcore Ethernet using a BMAP is studied in [17]. Under the assumption that a BMAP has a tree structure, rate matrices of a BMAP is estimated from traffic data by the EM algorithm. And the stationary probability is computed by the spectral method in [13]. Recently traffic measured by Wide Project is modeled by a BMAP without assuming its structure in [18]. The stationary probability of the queue length of a BMAP/D/1 queue is analytically derived by the spectral method given in this paper. It is found that the stationary probability of the analytical model is in good agreement with that of simulation driven from raw traffic. A BMAP seems to be a good model describing dynamics of IP traffic well.

We consider the algorithm of the spectral method for calculating the stationary probabilities of the queue length of a BMAP/G/1 queue just after service completion epochs and at arbitrary time. This algorithm can also be applied to other queueing model whose input process is formulated as a BMAP. The spectral method of the stationary probability of the queue length of a MAP/D/N is studied in [12], where Newton's method is proposed to calculate the zeros. However, applying the modified D-K method to a MAP/D/N queue we efficiently obtain the boundary vector. And the spectral method for calculating the stationary probability of a nonpreemptive priority queue with two classes of customers is given in [15]. In this case the boundary vector and the boundary generating function are efficiently calculated by the modified D-K method and the joint probability of two queue lengths of two classes are calculated by the inverse discrete two-dimensional FFT for the probability generating function.

In Section 2, definitions are enumerated. In Section 3, fundamental results for eigenvalues of the $z$-transform of rate matrices are proven. In Section 4 , it is proved in Theorem 4.3 that there are $M$ zeros of the determinant on a disk in the complex plane. And from the $M$ zeros and the corresponding null vectors, the vector $\boldsymbol{g}$ is derived in Theorems 4.4-4.5. In Section 5, for the calculation of all zeros on the disk, the modified D-K method with a double for-loop is introduced. In Section 6, for numerical examples, zeros are calculated by the modified D-K method and the stationary probabilities are obtained by the Fourier inversion transform of the vector generating function. In Appendix computing algorithms for the stationary probabilities just after service completion epochs and at arbitrary time 
are given.

\section{Definitions}

We define $\mathbf{0}=(0, \ldots, 0)$ and $\boldsymbol{e}=(1, \ldots, 1)^{\boldsymbol{T}}$, where the symbol $\boldsymbol{T}$ is the transposition of a matrix. Let $I$ be the identity matrix. We consider a batch Markovian arrival process whose state space of the underlying process is $\{i: i=1, \ldots, M\}$. Let $D_{k}=\left(d_{i, j}^{(k)}\right)(i, j=$ $1,2, \ldots, M)$ be the $M \times M$ matrix, where $d_{i, j}^{(k)}$ is phase transition rate from $i$ to $j$ with an arrival of batch size $k(k=1,2, \ldots)$. Let $D_{0}=\left(d_{i, j}^{(0)}\right)(i, j=1,2, \ldots, M)$ be the $M \times M$ matrix, where $d_{i, j}^{(0)}(i \neq j)$ is phase transition rate from $i$ to $j$ without arrival and

$$
d_{i, i}^{(0)}=-\left[\sum_{k=1}^{\infty} d_{i, i}^{(k)}+\sum_{j \neq i}^{M} \sum_{k=0}^{\infty} d_{i, j}^{(k)}\right] .
$$

Let $\delta=\max _{i}\left(-d_{i, i}^{(0)}\right)$. As usual, assume that for $|z| \leq 1$,

$$
D(z)=\sum_{k=0}^{\infty} D_{k} z^{k}=\left(d_{i, j}(z)\right)=\left(\sum_{k=0}^{\infty} d_{i, j}^{(k)} z^{k}\right)
$$

is analytic and that $D=D(1)=\sum_{k=0}^{\infty} D_{k}$ is the irreducible infinitesimal generator of the underlying process. And let $\boldsymbol{\pi}=\left(\pi_{1}, \pi_{2}, \ldots, \pi_{M}\right)$ be the stationary probability vector of $D$ $(\boldsymbol{\pi} D=\mathbf{0}$ and $\boldsymbol{\pi} \boldsymbol{e}=1)$. The mean arrival rate of customers is given by $\lambda=\boldsymbol{\pi} D^{\prime}(1) \boldsymbol{e}=$ $\boldsymbol{\pi} \sum_{k=1}^{\infty} k D_{k} \boldsymbol{e}$.

Let $H(t)$ be a distribution function of the service time with the mean $1 / \mu$. For simplicity, we set $H(0)=0$. Let $h(\alpha)=\int_{0}^{\infty} e^{\alpha t} d H(t)$ denote the moment generating function of $H(t)$. Let $A_{k}$ be the matrix whose $(i, j)$ element is the phase transition probability from $i$ to $j$ with an arrival of size $k$ during a service time. Then $A(z)=\sum_{k=0}^{\infty} A_{k} z^{k}=\int_{0}^{\infty} e^{D(z) t} d H(t)$. Assume that the traffic intensity $\rho=\lambda / \mu<1$.

\section{Eigenvalues of $D(z)$}

Let $\alpha_{i}(z)(i=1, \ldots, M)$ be the $i$ th eigenvalue of $D(z)$. Let

$$
\boldsymbol{u}_{i}(z)=\left(u_{i, 1}(z), \ldots, u_{i, j}(z), \ldots, u_{i, M}(z)\right),
$$

and

$$
\boldsymbol{v}_{i}(z)=\left(v_{1, i}(z), \ldots, v_{j, i}(z), \ldots, v_{M, i}(z)\right)^{\boldsymbol{T}},
$$

be the corresponding left and right eigenvectors $\left(\boldsymbol{u}_{i}(z) D(z)=\alpha_{i}(z) \boldsymbol{u}_{i}(z)\right.$ and $D(z) \boldsymbol{v}_{i}(z)=$ $\left.\alpha_{i}(z) \boldsymbol{v}_{i}(z)\right)$ normalized as $U(z) V(z)=I$ with

$$
U(z)=\left(\begin{array}{c}
\boldsymbol{u}_{1}(z) \\
\vdots \\
\boldsymbol{u}_{M}(z)
\end{array}\right) \text { and } V(z)=\left(\boldsymbol{v}_{1}(z), \ldots, \boldsymbol{v}_{M}(z)\right)
$$

Particularly, $\alpha_{1}(1)=0, \boldsymbol{u}_{1}(1)=\boldsymbol{\pi}$ and $\boldsymbol{v}_{1}(1)=\boldsymbol{e}$. We assume that in later discussions all eigenvalues are simple. The corresponding left and right eigenvectors are efficiently calculated.

Assumption 1 For $z$ on the unit circle $(|z|=1)$, all eigenvalues $\alpha_{i}(z)$ are simple. 
Suppose $\alpha_{i}(\widetilde{z})$ is simple. The characteristic function $\operatorname{det}(\alpha I-D(z))$ is a polynomial in $\alpha$ of degree $M$ and $d_{i, j}(z)$ is analytic for each $i$ and $j$. It follows from the theorem on implicit functions that $\alpha_{i}(z)$ is analytic in a neighborhood of $z=\widetilde{z}$ (see [16]).

We use the matrix norm $\|D(z)\|_{\infty}$ defined as

$$
\|D(z)\|_{\infty}=\max _{i} \sum_{j=1}^{M}\left|d_{i j}(z)\right| .
$$

Proposition 3.1 For any $z$ on the unit disk, a region $\Omega$ of eigenvalues $\alpha_{i}(z)$ is given by the disk of radius $\delta$ with the center $-\delta$ in the complex plane. That is,

$$
\alpha_{i}(z) \in \Omega \equiv\{\alpha:|\alpha+\delta| \leq \delta\}
$$

Proof. If $\alpha$ is an eigenvalue of $D(z)$,

$$
\begin{aligned}
0 & =\operatorname{det}(\alpha I-D(z)) \\
& =\delta^{M} \operatorname{det}\left(\left(\frac{\alpha}{\delta}+1\right) I-\left(\frac{D(z)}{\delta}+I\right)\right) .
\end{aligned}
$$

Since all terms in the bracket in the right hand side of (2.1) are nonnegative and $\delta+d_{i, i}^{(0)} \geq 0$, for $\frac{1}{\delta} D(z)+I$, the $i$ th row sum of absolute values is

$$
\begin{aligned}
& \frac{1}{\delta}\left(\left|\delta+d_{i, i}(z)\right|+\sum_{j \neq i}^{M}\left|d_{i, j}(z)\right|\right) \\
\leq & \frac{1}{\delta}\left(\left|\delta+d_{i, i}^{(0)}\right|+\left|\sum_{k=1}^{\infty} d_{i, i}^{(k)} z^{k}\right|+\sum_{j \neq i}^{M}\left|\sum_{k=0}^{\infty} d_{i, j}^{(k)} z^{k}\right|\right) \\
\leq & \frac{1}{\delta}\left(\delta+d_{i, i}^{(0)}+\sum_{k=1}^{\infty} d_{i, i}^{(k)}+\sum_{j \neq i}^{M} \sum_{k=0}^{\infty} d_{i, j}^{(k)}\right) \\
= & 1 .
\end{aligned}
$$

The spectral radius of $\frac{1}{\delta} D(z)+I$ is

$$
\left|\frac{\alpha}{\delta}+1\right| \leq\left\|\frac{1}{\delta} D(z)+I\right\|_{\infty} \leq 1,
$$

(see [24]). This leads the conclusion.

We have the next proposition.

Proposition 3.2 Suppose $\alpha_{i}(\zeta)$ is simple. Then

$$
\left.\frac{d}{d z} \alpha_{i}(z)\right|_{z=\zeta}=\boldsymbol{u}_{i}(\zeta) D^{\prime}(\zeta) \boldsymbol{v}_{i}(\zeta)
$$

Proof. From the assumption, an eigenvalue $\alpha_{i}(z)$ is analytic in a neighborhood of $z=\zeta$. It is also proved that there exists the corresponding analytic left (res. right) eigenvector $\boldsymbol{u}_{i}(z)$ $\left(\right.$ res. $\left.\boldsymbol{v}_{i}(z)\right)$ in a neighborhood of $z=\zeta$ (see [16]). Therefore, we have

$$
\begin{aligned}
& \alpha_{i}(z)=\alpha_{i}(\zeta)+(z-\zeta) \alpha_{i}^{\prime}(\zeta)+o(|z-\zeta|), \\
& \boldsymbol{u}_{i}(z)=\boldsymbol{u}_{i}(\zeta)+(z-\zeta) \boldsymbol{u}_{i}^{\prime}(\zeta)+o(|z-\zeta|), \\
& \boldsymbol{v}_{i}(z)=\boldsymbol{v}_{i}(\zeta)+(z-\zeta) \boldsymbol{v}_{i}^{\prime}(\zeta)+o(|z-\zeta|),
\end{aligned}
$$


in a neighborhood of $z=\zeta$. Substituting the above expansions of $\alpha_{i}(z)$ and $\boldsymbol{u}_{i}(z)$ into $\boldsymbol{u}_{i}(z) D(z)=\alpha_{i}(z) \boldsymbol{u}_{i}(z)$ and comparing the coefficients of $z-\zeta$ we have

$$
\boldsymbol{u}_{i}^{\prime}(\zeta)\left(D(\zeta)-\alpha_{i}(\zeta) I\right)=\boldsymbol{u}_{i}(\zeta)\left(D^{\prime}(\zeta)-\alpha_{i}^{\prime}(\zeta) I\right)
$$

By postmultiplying $\boldsymbol{v}_{i}(\zeta)$, we have

$$
\left.\frac{d}{d z} \alpha_{i}(z)\right|_{z=\zeta}=\boldsymbol{u}_{i}(\zeta) D^{\prime}(\zeta) \boldsymbol{v}_{i}(\zeta)
$$

because the left hand side is equal to 0 and $\boldsymbol{u}_{i}(\zeta) \boldsymbol{v}_{i}(\zeta)=1$.

\section{Spectral Method for the Vector $g$}

Let $\boldsymbol{p}_{k}=\left(p_{k, 1}, \ldots, p_{k, M}\right)(k \geq 0)$ be the $M$-dimensional row vector whose $i$ th entry $p_{k, i}$ is the stationary joint probability that just after service completion epochs, the arrival phase is $i$ and the number of customers in the system is $k$. Then $\boldsymbol{p}=\left(\boldsymbol{p}_{0}, \boldsymbol{p}_{1}, \ldots\right)$ is the stationary probability vector just after service completion epochs. Define $\boldsymbol{p}(z)=\sum_{k=0}^{\infty} \boldsymbol{p}_{k} z^{k}$. As in Neuts [11] let $G$ and $\boldsymbol{g}$ be the phase transition stochastic matrix of the first passage time from the level $k+1$ to $k$ and its invariant probability vector $(\boldsymbol{g} G=\boldsymbol{g}, \quad \boldsymbol{g} \boldsymbol{e}=1)$, respectively. In Lucantoni [7], $\boldsymbol{p}(z)$ is given by

$$
\boldsymbol{p}(z)=\lambda^{-1}(1-\rho) \boldsymbol{g} D(z) A(z)(z I-A(z))^{-1} .
$$

Similarly, let $\boldsymbol{q}_{k}=\left(q_{k, 1}, \ldots, q_{k, M}\right)(k \geq 0)$ be the $M$-dimensional row vector whose $i$ th entry $q_{k, i}$ is the stationary joint probability that the arrival phase is $i$ and the number of customers in the system is $k$ at arbitrary time. For the stationary probability vector $\boldsymbol{q}=\left(\boldsymbol{q}_{0}, \boldsymbol{q}_{1}, \ldots\right)$ at arbitrary time, the vector generating function $\boldsymbol{q}(z)=\sum_{k=0}^{\infty} \boldsymbol{q}_{k} z^{k}$ is given by

$$
\boldsymbol{q}(z)=(1-\rho)(z-1) \boldsymbol{g} A(z)(z I-A(z))^{-1}
$$

in $[7]$.

In this section we consider a spectral method based on eigenvalues for calculating the probability vector of $\boldsymbol{g}$. In Gail et al. [4] and [5], for an M/G/1 type Markov chain, the number of zeros of $\operatorname{det}(z I-A(z))$ on the unit disk is studied. In particular, under the ergodic condition, there are $M$ zeros on the disk and $G$ is derived by the zeros and the corresponding null vectors. Since the infinitesimal generator $D$ is irreducible and $\rho<1$, the BMAP/G/1 we consider is an ergodic M/G/1 type Markov chain. Then we have the following theorem.

Theorem 4.1 ([4], [5]) There are $M$ zeros of

$$
\operatorname{det}(z I-A(z))=0
$$

on the unit disk $(|z| \leq 1)$ counting multiplicities. And 1 is a simple zero and there are $M-1$ zeros on the open unit disk $(|z|<1)$.

The next theorem is proved in Lucantoni ([7]).

Theorem 4.2 ([r] Let $D[G]$ be the infinitesimal generator of a Markov process obtained by excising the busy period defined by $D[G]=\sum_{k=0}^{\infty} D_{k} G^{k}$. Then

$$
G=\int_{0}^{\infty} e^{D[G] t} d H(t)
$$


From the above two theorems, we will prove the next theorem. Let $\gamma_{i}(i=1, \ldots, M)$ and $\boldsymbol{\eta}_{i}$ be the $i$ th eigenvalue of $D[G]$ and the corresponding right eigenvector with $\gamma_{1}=0$ and $\boldsymbol{\eta}_{1}=\boldsymbol{e}$. Denote $z_{i}=h\left(\gamma_{i}\right)(i=1, \ldots, M)$.

Theorem 4.3 1. $z_{i}(i=1, \ldots, M)$ is an eigenvalue of $G$. And $\boldsymbol{\eta}_{i}$ is the corresponding eigenvector of $G$.

2. $z_{i}(i=1, \ldots, M)$ are the $M$ zeros of $\operatorname{det}(z I-A(z))$ in Theorem 4.1. $\boldsymbol{\eta}_{i}$ is the corresponding right null vector of $z_{i} I-A\left(z_{i}\right)$.

3. Counting multiplicities, there are $M$ zeros of

$$
\operatorname{det}(\alpha I-D(h(\alpha)))
$$

in $\Omega . \gamma_{i}(i=1, \ldots, M)$ and $\boldsymbol{\eta}_{i}$ are the $M$ zeros of (4.5) in $\Omega$ and the corresponding right null vector of

$$
\gamma_{i} I-D\left(h\left(\gamma_{i}\right)\right)
$$

respectively.

Proof. 1. From (4.4) $D[G]$ and $G$ have the common right eigenvectors $\boldsymbol{\eta}_{i}(i=1, \ldots, M)$. Then $h\left(\gamma_{i}\right)(i=1, \ldots, M)$ are the $M$ eigenvalues of $G$ counting multiplicity.

2. Postmultiplying (4.4) by $\boldsymbol{\eta}_{i}$ we get

$$
\begin{aligned}
h\left(\gamma_{i}\right) \boldsymbol{\eta}_{i} & =\int_{0}^{\infty} e^{D\left(h\left(\gamma_{i}\right)\right) t} d H(t) \boldsymbol{\eta}_{i} \\
& =A\left(h\left(\gamma_{i}\right)\right) \boldsymbol{\eta}_{i},
\end{aligned}
$$

because $A(z)=\int_{0}^{\infty} e^{D(z) t} d H(t)$. This implies that

$$
\operatorname{det}\left(h\left(\gamma_{i}\right) I-A\left(h\left(\gamma_{i}\right)\right)\right)=0 .
$$

Therefore, $z_{i}=h\left(\gamma_{i}\right)(i=1, \ldots, M)$ is the $M$ zeros of (4.3) in Theorem 4.1 counting multiplicities. And $\boldsymbol{\eta}_{i}$ is the corresponding right null vector of $h\left(\gamma_{i}\right) I-A\left(h\left(\gamma_{i}\right)\right)$.

3. Postproducting $\gamma_{i} I-D[G]$ by $\boldsymbol{\eta}_{i}$, we see that $\left(\gamma_{i} I-D\left(h\left(\gamma_{i}\right)\right)\right) \boldsymbol{\eta}_{i}=\left(\gamma_{i} I-D[G]\right) \boldsymbol{\eta}_{i}=0$. So, $\gamma_{i}$ is an eigenvalue of $D\left(h\left(\gamma_{i}\right)\right)$. Then $\gamma_{i}(i=1, \ldots, M)$ are zeros of (4.5) in $\Omega$. And there are at least $M$ zeros of (4.5) in $\Omega$ counting multiplicity.

Suppose that counting multiplicities there is another zero of $(4.5)$ in $\Omega$, say $\operatorname{det}(\gamma I-$ $D(h(\gamma)))=0$. Then $h(\gamma)$ is on the unit disk and $\operatorname{det}(h(\gamma) I-A(h(\gamma)))=0$, that is, $h(\gamma)$ is a zero of (4.3) in Theorem 4.1. This contradicts Theorem 4.1. Therefore, $\gamma_{i}(i=1, \ldots, M)$ are the $M$ zeros of (4.5) in $\Omega$ and $\boldsymbol{\eta}_{i}$ is the right null vector of $\gamma_{i} I-D\left(h\left(\gamma_{i}\right)\right)$. The proof is completed.

We set the following assumption.

Assumption 2 All the $M$ zeros $\gamma_{i}(i=1, \ldots, M)$ of $\operatorname{det}(\alpha I-D(h(\alpha)))$ in $\Omega$ are simple.

Define the matrix $Y$ as $Y=\left(\boldsymbol{\eta}_{1}, \ldots, \boldsymbol{\eta}_{M}\right)$. From Assumption $2 Y$ is nonsingular. From Theorem 4.2, we have the diagonal representation of $G$.

\section{Theorem 4.4}

$$
G=Y\left(\begin{array}{ccc}
z_{1} & & \\
& \ddots & \\
& & z_{M}
\end{array}\right) Y^{-1} .
$$


The matrix $G$ calculated by (4.8) should be stochastic. This property is useful to check the precision of numerically calculated zero points and the corresponding null vectors. From $\boldsymbol{g} G=\boldsymbol{g}$ and $\boldsymbol{g} \boldsymbol{e}=1, \boldsymbol{g}$ is calculated. If we need $\boldsymbol{g}$ in (4.1) not $G$, we directly obtain the vector $\boldsymbol{g}$ as follows.

Theorem 4.5 The vector $\boldsymbol{g}$ is given by

$$
\boldsymbol{g}=\boldsymbol{e}_{1} Y^{-1}
$$

where $\boldsymbol{e}_{1}=(1,0, \ldots, 0)$ is the first unit column vector.

Proof. By postmultiplyion of $\boldsymbol{p}\left(z_{i}\right)\left(z_{i} I-A\left(z_{i}\right)\right)=\lambda^{-1}(1-\rho) \boldsymbol{g} D\left(z_{i}\right) A\left(z_{i}\right)$ by $\boldsymbol{\eta}_{i}$, from $\left(z_{i} I-A\left(z_{i}\right)\right) \boldsymbol{\eta}_{i}=\mathbf{0}, D\left(z_{i}\right) \boldsymbol{\eta}_{i}=\gamma_{i} \boldsymbol{\eta}_{i}$ and $\gamma_{i} \neq 0(i=2, \ldots, M)$, we have

$$
\boldsymbol{g}_{i}=0 \quad i=2, \ldots, M .
$$

And $\boldsymbol{g} \boldsymbol{\eta}_{1}=\boldsymbol{g} \boldsymbol{e}=1$. The proof is completed.

It is hard to find all the distinct $M$ zeros $z_{i}$ of (4.3) on the unit disk. By the virtue of Theorem 4.3 if we get distinct $M$ zeros $\gamma_{i}(i=1, \ldots, M)$ of $(4.5)$ in $\Omega, z_{i}=h\left(\gamma_{i}\right)$ $(i=1, \ldots, M)$ are all the distinct $M$ zeros $z_{i}$ of $(4.3)$ on the unit disk. Our next problem is how to calculate all the $M$ zeros of (4.5) in $\Omega$. In general, the calculations of matrices $A_{k}$ require a large memory and a large computational time if a BMAP contains a large batch size. For our method, $\boldsymbol{g}$ is obtained without $A_{k}$. The spectral method for calculations of the stationary vectors $\boldsymbol{p}$ and $\boldsymbol{q}$ are given in Appendix.

\section{The Modified Durand-Kerner Method}

The Durand-Kerner method (the D-K method) is known as an iterative method for calculation of all zeros of a polynomial, simultaneously. Let $P(\alpha)$ be a polynomial of degree $M$ whose coefficient of $\alpha^{M}$ is 1 and zeros are $\gamma_{i}(i=1, \ldots, M)$. We have

$$
P(\alpha)=\sum_{i=0}^{M} a_{i} \alpha^{i}=\prod_{i=1}^{M}\left(\alpha-\gamma_{i}\right)
$$

where $a_{i}(i=0, \ldots, M)$ are coefficients of a polynomial with $a_{M}=1$. Suppose that as the $\nu$ th iterative approximation of $\gamma_{1}, \ldots, \gamma_{M}$ we have $M$ distinct $\alpha_{1}^{(\nu)}, \ldots, \alpha_{M}^{(\nu)}$. By defining $\Delta \alpha_{i}^{(\nu)} \equiv \gamma_{i}-\alpha_{i}^{(\nu)}$, the expansion of $P(\alpha)$ is written as

$$
P(\alpha)=\prod_{i=1}^{M}\left(\alpha-\alpha_{i}^{(\nu)}\right)-\sum_{i=1}^{M} \Delta \alpha_{i}^{(\nu)} \prod_{j=1, j \neq i}^{M}\left(\alpha-\alpha_{j}^{(\nu)}\right)+\cdots .
$$

Dropping all terms of $\Delta \alpha_{i}$ higher than the first and substituting $\alpha_{i}^{(\nu)}$ to $P(\alpha)$, we have

$$
P\left(\alpha_{i}^{(\nu)}\right) \approx-\Delta \alpha_{i}^{(\nu)} \prod_{j=1, j \neq i}^{M}\left(\alpha_{i}^{(\nu)}-\alpha_{j}^{(\nu)}\right)
$$

that is,

$$
\Delta \alpha_{i}^{(\nu)} \approx-\frac{P\left(\alpha_{i}^{(\nu)}\right)}{\prod_{j=1, j \neq i}^{M}\left(\alpha_{i}^{(\nu)}-\alpha_{j}^{(\nu)}\right)} .
$$


From the $\nu$ th approximation, the $(\nu+1)$ st approximation of $\gamma_{i}$ is defined as

$$
\alpha_{i}^{(\nu+1)}=\alpha_{i}^{(\nu)}-\frac{P\left(\alpha_{i}^{(\nu)}\right)}{\prod_{j=1, j \neq i}^{M}\left(\alpha_{i}^{(\nu)}-\alpha_{j}^{(\nu)}\right)} .
$$

This iteration is known as the D-K method ([1], [6], [22] and [25]). It is proved that the $\mathrm{D}-\mathrm{K}$ method is a locally quadratic convergence if all zeros are simple. And several improved methods which are a cubic convergence have been studied.

In this calculation, the property that all zeros of a polynomial are calculated simultaneously is useful for our problem. By using the D-K method we consider to obtain all the $M$ zeros $\gamma_{i}(i=1, \ldots, M)$ of $(4.5)$ in $\Omega$, simultaneously. It should be noted that for the equation (4.5) in general, there are infinitely many zeros which we need not use. We consider a modification of the D-K method to fit our problem. Since from Assumption $2 \gamma_{i}$ $(i=1, \ldots, M)$ are simple and $\operatorname{det}(\alpha I-D(h(\alpha)))$ is analytic in $\Omega$, there exists a function $\phi(\alpha)$ such that $\phi(\alpha)$ is unknown but analytic in $\Omega$ and is not equal to 0 for $\alpha \in \Omega$, and $f(\alpha) \equiv \operatorname{det}(\alpha I-D(h(\alpha)))$ is represented as

$$
f(\alpha)=\phi(\alpha) \prod_{j=1}^{M}\left(\alpha-\gamma_{j}\right) .
$$

If $\alpha$ is in a neighborhood of $\gamma_{i}$, we may approximate as

$$
\begin{aligned}
P(\alpha) & =\prod_{j=1}^{M}\left(\alpha-\gamma_{j}\right) \\
& \approx \frac{f(\alpha)}{\phi\left(\gamma_{i}\right)} .
\end{aligned}
$$

From (5.1), we define an iteration given by

$$
\alpha_{i}^{(\nu+1)}=\alpha_{i}^{(\nu)}-\frac{\operatorname{det}\left(\alpha_{i}^{(\nu)} I-D\left(h\left(\alpha_{i}^{(\nu)}\right)\right)\right)}{\phi\left(\gamma_{i}\right) \prod_{j=1, j \neq i}^{M}\left(\alpha_{i}^{(\nu)}-\alpha_{j}^{(\nu)}\right)} .
$$

In the next proposition we derive $\phi\left(\gamma_{i}\right)$.

Proposition 5.1 Suppose $\gamma_{i}$ is the $l(i)$ th eigenvalue of $D\left(z_{i}\right)$, i.e. $\gamma_{i}=\alpha_{l(i)}\left(z_{i}\right)$. Then we have

$$
\phi\left(\gamma_{i}\right)=\left(1-\alpha_{l(i)}^{\prime}\left(z_{i}\right) h^{\prime}\left(\gamma_{i}\right)\right) \frac{\prod_{j=1, j \neq l(i)}^{M}\left(\gamma_{i}-\alpha_{j}\left(z_{i}\right)\right)}{\prod_{j=1, j \neq i}^{M}\left(\gamma_{i}-\gamma_{j}\right)},
$$

where $\alpha_{j}\left(z_{i}\right)$ is the $j$ th eigenvalue of $D\left(z_{i}\right)$ and $\alpha_{l(i)}^{\prime}\left(z_{i}\right)=\boldsymbol{u}_{l(i)}\left(z_{i}\right) D^{\prime}\left(z_{i}\right) \boldsymbol{v}_{l(i)}\left(z_{i}\right)$ is given in Proposition 3.2.

Proof. Since the $j$ th eigenvalue of $\alpha I-D(h(\alpha))$ is $\alpha-\alpha_{j}(h(\alpha))$ and the determinant of a matrix is the product of eigenvalues, we have

$$
f(\alpha)=\prod_{j=1}^{M}\left(\alpha-\alpha_{j}(h(\alpha))\right) .
$$

The function $f(\alpha)$ has simple zeros $\gamma_{i}$ of $\operatorname{det}(\alpha I-D(h(\alpha)))$. Put $z_{i}=h\left(\gamma_{i}\right)$. By differentiating $f(\alpha)$ at $\alpha=\gamma_{i}$, from Proposition 3.2 we have

$$
\begin{aligned}
f^{\prime}\left(\gamma_{i}\right) & =\sum_{k=1}^{M}\left(1-\alpha_{k}^{\prime}\left(h\left(\gamma_{i}\right)\right) h^{\prime}\left(\gamma_{i}\right)\right) \prod_{j=1, j \neq k}^{M}\left(\gamma_{i}-\alpha_{j}\left(h\left(\gamma_{i}\right)\right)\right) \\
& =\sum_{k=1}^{M}\left(1-\alpha_{k}^{\prime}\left(z_{i}\right) h^{\prime}\left(\gamma_{i}\right)\right) \prod_{j=1, j \neq k}^{M}\left(\gamma_{i}-\alpha_{j}\left(z_{i}\right)\right),
\end{aligned}
$$


where $\alpha_{k}^{\prime}\left(z_{i}\right)=\boldsymbol{u}_{k}\left(z_{i}\right) D^{\prime}\left(z_{i}\right) \boldsymbol{v}_{k}\left(z_{i}\right)$. For $k \neq l(i)$ the product of the right hand side is 0 because $\gamma_{i}=\alpha_{l(i)}\left(z_{i}\right)$ for $j=l(i)$. Therefore,

$$
f^{\prime}\left(\gamma_{i}\right)=\left(1-\alpha_{l(i)}^{\prime}\left(z_{i}\right) h^{\prime}\left(\gamma_{i}\right)\right) \prod_{j=1, j \neq l(i)}^{M}\left(\gamma_{i}-\alpha_{j}\left(z_{i}\right)\right) .
$$

On the other hand by differentiating (5.2) at $\alpha=\gamma_{i}$ we have

$$
f^{\prime}\left(\gamma_{i}\right)=\phi^{\prime}\left(\gamma_{i}\right) \prod_{j=1}^{M}\left(\gamma_{i}-\gamma_{j}\right)+\phi\left(\gamma_{i}\right) \sum_{k=1}^{M} \prod_{j=1, j \neq k}^{M}\left(\gamma_{i}-\gamma_{j}\right) .
$$

The first term in the right hand side is 0 because $\prod_{j=1}^{M}\left(\gamma_{i}-\gamma_{j}\right)=0$. And

$$
\prod_{j=1, j \neq k}^{M}\left(\gamma_{i}-\gamma_{j}\right)=0 \quad \text { if } \quad k \neq i
$$

Then

$$
f^{\prime}\left(\gamma_{i}\right)=\phi\left(\gamma_{i}\right) \prod_{j=1, j \neq i}^{M}\left(\gamma_{i}-\gamma_{j}\right)
$$

Comparing (5.6) and (5.7), we get (5.5).

There are $M$ zeros of $\operatorname{det}(\alpha I-D(h(\alpha)))$ in $\Omega$ but in general $\operatorname{det}(\alpha I-D(h(\alpha)))$ has infinite unnecessary zeros on the right-half complex plane $\Omega^{*}=\{\alpha \mid \Re(\alpha)>0\}$. For unsuitable initial values an iterative sequence converges to an unnecessary zero on $\Omega^{*}$. This situation is quite different from the problem for calculating zeros of a polynomial by the Durand-Kerner method. To overcome this difficulty we propose a double for-loop iteration.

Suppose $\mu_{s}$ be a decreasing sequence of service rates $\left(\mu_{0}=\infty>\mu_{1}>\ldots>\mu_{K}=\mu\right)$. Define the sequence of distribution functions of the service time as $H^{(s)}(t)=H\left(\frac{\mu_{s}}{\mu} t\right)$. For each $H^{(s)}(t)$, let $\gamma_{i}^{(s)}(i=1, \ldots, M)$ be the zeros of $\operatorname{det}\left(\alpha I-D\left(h^{(s)}(\alpha)\right)\right)$ in $\Omega$, where $h^{(s)}(\alpha)=\int_{0}^{\infty} e^{\alpha t} d H^{(s)}(t)$. In the case of $s=0, \gamma_{i}^{(0)}$ is an eigenvalue of $D(1)$ and $\gamma_{i}^{(0)}=\alpha_{i}(1)$. And $h^{(0)}(\alpha) \equiv 1$ implies that $h^{(0)}(\alpha)^{\prime} \equiv 0$ in $(5.5)$. So $\phi^{(0)}\left(\gamma_{i}^{(0)}\right) \equiv 1$.

In order to calculate $\gamma_{i}^{(s)}(i=1, \ldots, M)$ for $s=1, \ldots, K$ we propose the iteration with initial values $\gamma_{i}^{(s-1)}(i=1, \ldots, M)$. The intermediate zeros $\gamma_{i}^{(s)}(s=1, \ldots, K-1, i=$ $1, \ldots, M)$ are need not to be numerically precise values. For $s<K$ we set the number $N_{1}$ of iterations to a small number, e.g. $N_{1}=5$ or 10 . However, the zeros $\gamma_{i}^{(K)}(i=1, \ldots, M)$ should be numerically precise values. For $s=K$ we set the number $N_{2}$ of iterations to a large number, e.g. $N_{2}=50$ or 100 . This algorithm is summarized as

Algorithm(the modified D-K method)

1. Calculate all the eigenvalues $\alpha_{i}(1)$ of $D(1)$. Set $\gamma_{i}^{(0)}=\alpha_{i}(1), \phi^{(0)}\left(\gamma_{i}^{(0)}\right)=1, s=1, \nu=1$. If $K>1$ then set $N=N_{1}$ else $N=N_{2}$.

2. Calculate

$$
\alpha_{i}^{(s, \nu+1)}=\alpha_{i}^{(s, \nu)}-\frac{\operatorname{det}\left(\alpha_{i}^{(s, \nu)} I-D\left(h^{(s)}\left(\alpha_{i}^{(s, \nu)}\right)\right)\right)}{\prod_{j=1, j \neq i}^{M}\left(\alpha_{i}^{(s, \nu)}-\alpha_{j}^{(s, \nu)}\right)} \cdot \frac{1}{\phi^{(s-1)}\left(\gamma_{i}^{(s-1)}\right)},
$$

for $i=1, \ldots, M$. 
3. If $\nu<N$ then $\operatorname{set} \nu=\nu+1$ and go to Step 2 .

4. Set $\gamma_{i}^{(s)}=\alpha_{i}^{(s, N+1)}$ and calculate $\phi^{(s)}\left(\gamma_{i}^{(s)}\right)$ by (5.5). If $s \leq K-2$ then set $\nu=1$ and $s=s+1$ and go to Step 2. If $s=K-1$ then set $\nu=1, s=K$ and $N=N_{2}$ and go to Step 2.

5. Set $\gamma_{i}=\gamma_{i}^{(K)}$ and calculate the right null vector $\boldsymbol{\eta}_{i}$ of $\gamma_{i} I-D\left(h\left(\gamma_{i}\right)\right)$ with the max norm $\left\|\boldsymbol{\eta}_{i}\right\|_{\infty}=1$.

6. End.

Remark 1. It is assumed that for the conventional D-K method in (5.1) the coefficient $a_{M}$ of $\alpha^{M}$ is 1 . Since our problem is calculating only the zeros on $\Omega$, there is an unknown analytic function $\phi(\alpha)$ in (5.2). For the modified D-K method in (5.8), $\phi^{(s-1)}\left(\gamma_{i}^{(s-1)}\right)$ is an approximation of the unknown non-zero function $\phi(\alpha)$. Comparing a method with $\phi^{(s)}(\alpha) \equiv$ 1 we get a good convergence of the iterative sequence derived by (5.8).

Remark 2. For a fixed sufficiently small number $\epsilon>0$, the accuracy of $\gamma_{i}$ is checked by

$$
\max _{i=1, \ldots, M}\left\|\left(\gamma_{i} I-D\left(h\left(\gamma_{i}\right)\right)\right) \boldsymbol{\eta}_{i}\right\|_{\infty}<\epsilon .
$$

This formula also can be used to the stopping criterion instead of the fixed number $N_{2}$.

Remark 3. In the numerical examples of the next section, the sequence $\left\{\mu_{s} \mid s=1, \ldots, K\right\}$ is defined as $\mu^{(s)}=K \mu / s$. In this case we have $\rho^{(s)} \equiv \lambda / \mu^{(s)}=s \rho / K$.

\section{Numerical Examples}

In this section, we implement two numerical experiments. The stationary probabilities of the number of customers just after service completion epochs and at arbitrary time are calculated by two computational steps. The first is the computation for the zeros $\gamma_{i}$ of $\operatorname{det}(\alpha I-D(h(\alpha)))$ by the modified D-K method in Section 5. From Theorems 4.3-4.5, the vector $\boldsymbol{g}$ is obtained. The second step is to calculate the stationary probability $\boldsymbol{p}_{n}$ and $\boldsymbol{q}_{n}$ by using a spectral method based on the fast Fourier inversion transform in Appendix. All calculations are performed for double precision in DECIMAL BASIC on the personal computer (CPU 3.06GHz, RAM 512MB).

\section{Model 1}

In order to check the efficiency of our algorithm we require the following three properties to a BMAP for a numerical example:

1. a BMAP is bursty,

2. there are complex zeros $\gamma_{i}$,

3. the maximum batch size is large.

It is known that the model introduced in [21] is bursty but for this tree-structured model, all $\gamma_{i}$ are real and all arrivals are single (a MAP). We modify this model. There are the root phase 1 and $C$ cyclic leaves with 3 phases $(M=3 C+1)$ as illustrated in Figure 1 . From these cyclic leaves complex zeros $\gamma_{i}$ are derived. The phases in $c$ th leaf are numbered as $3 c-1,3 c$ and $3 c+1$. For each $i$ and $j(i, j=1, \ldots, M)$, the phase transition rates without arrival and the arrival rates without transition are given by

$$
\begin{cases}d_{1,3 c-1}^{(0)}=\frac{c}{a}, & d_{3 c-1,1}^{(0)}=c \\ d_{3 c-1,3 c}^{(0)}=d_{3 c, 3 c+1}^{(0)}=d_{3 c+1,3 c-1}^{(0)}=\frac{c}{a} \\ d_{3 c-1,3 c-1}^{\left(b^{c-1}\right)} \quad(c=1,2, \ldots, C), \\ d_{i, j}^{(k)}=0 \quad \text { (otherwise) }\end{cases}
$$

where $a$ is positive and $b$ is an integer. In the first line, transition rates from 1 to $3 c-1$ and from $3 c-1$ to 1 are given. By the second line, a cyclic phase transition in a leaf is represented. In the third line, arrival rates with batch size $b^{c-1}$ at $3 c-1$ are given. 
Table 1: Numerical results of $\alpha_{i}(1), \gamma_{i}\left(z_{i}\right), z_{i}$ and $\boldsymbol{g}$

\begin{tabular}{cccc}
\hline$\alpha_{i}(1)$ & $\gamma_{i}$ & $z_{i}$ & $g_{i}$ \\
\hline 0 & 0 & 1 & 0.16008 \\
\hline$-2.82857-0.39884 \boldsymbol{i}$ & -4.10388 & 0.81599 & 0.09885 \\
\hline$-2.82857+0.39884 \boldsymbol{i}$ & -2.15196 & 0.89885 & 0.11862 \\
\hline$-1.72423-0.52062 \boldsymbol{i}$ & $-1.90689-0.40443 \boldsymbol{i}$ & $0.90965-0.01823 \boldsymbol{i}$ & 0.13178 \\
\hline$-1.72423+0.52062 \boldsymbol{i}$ & $-1.90689+0.40443 \boldsymbol{i}$ & $0.90965+0.01823 \boldsymbol{i}$ & 0.07246 \\
\hline$-1.01265-0.22747 \boldsymbol{i}$ & $-1.03554-0.19982 \boldsymbol{i}$ & $0.94993-0.00940 \boldsymbol{i}$ & 0.09504 \\
\hline$-1.01265+0.22747 \boldsymbol{i}$ & $-1.03554+0.19982 \boldsymbol{i}$ & $0.94993+0.00940 \boldsymbol{i}$ & 0.10827 \\
\hline-6.18078 & -6.85028 & 0.71217 & 0.06649 \\
\hline-0.45866 & -0.53398 & 0.97388 & 0.07178 \\
\hline-0.22962 & -0.24940 & 0.98771 & 0.07657 \\
\hline
\end{tabular}

We set parameters as $C=3, M=10, a=2$ and $b=10$. For the deterministic service time with $\rho=0.5$ the stationary probabilities are calculated as followings. By the QR method we calculate eigenvalues $\alpha_{i}(1)$, where there are 4 real numbers and 3 pairs of mutually complex conjugate numbers. Using the modified $\mathrm{D}-\mathrm{K}$ method, for $K=1$ we calculate $\gamma_{i}$, where there are 6 real numbers and 2 pairs of mutually complex conjugate numbers. And from Theorems $4.3-4.5, z_{i}$ and $g_{i}$ are calculated. The 5 place numbers of these computational results in double precision are given in Table 1.

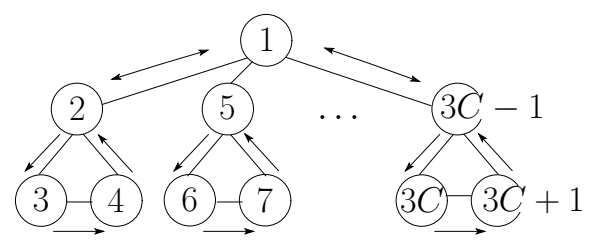

Figure 1: The phase transition

Next using Propositions 7.1-7.2 $\boldsymbol{p}_{n}$ and $\boldsymbol{q}_{n}$ are calculated by the Fourier inversion transform. Let $p_{n}=\boldsymbol{p}_{n} \boldsymbol{e}$ and $q_{n}=\boldsymbol{q}_{n} \boldsymbol{e}$ be the queue length probabilities just after service completion epochs and at arbitrary time, respectively. And $F(n)$ and $G(n)$ are the corresponding cumulative distribution functions. The computational results of $p_{n}, q_{n}, F(n)$ and $G(n)$ are listed in Table 2. Particularly, $p_{n}$ is illustrated in Figure 2. Since for arbitrary time $q_{0}=.5$ by Little's formula but $q_{n}<.006(n>0)$ with a long tail, it is hard to illustrate $q_{n}$ in one graph. This comes form the bursty BMAP whose batch sizes are $10^{c-1} c=1,2,3$. For service completion epochs $p_{0}-p_{1}, p_{9}-p_{10}$ and $p_{99}-p_{100}$ are nearly $3 \times 10^{-3}$, see Figure 2 and Table 2. For a small $n G(n)-F(n)$ is large but for a large $n q_{n} \approx .5 \times p_{n}$ from $\rho=.5$. For the first and second computational steps defined in the first paragraph of this section, the elapsed time is $1.9 \mathrm{sec}$. and $220 \mathrm{sec}$, respectively.

Setting parameters $[M=10, C=3, a=2, b=10, \rho=0.95]$ and $[M=31, C=10, a=$ $1.1, b=2, \rho=0.5]$, in two cases (i.e. heavy traffic and a large number of phases), we calculate zeros and the stationary probability $p_{k}$. In the second case, we set $a=1.1$ because for $a=2$ and $M=31$, the tail of the distribution function is too long. In both cases the stationary probabilities $p_{k}$ are calculated. We report $T_{1}, T_{2}, n^{*}$ in Table 3 , where $T_{i}$ is the time (sec.) needed for the $i$ th computational step $(i=1,2)$ and $n^{*}=\min \left\{n: F(n) \geq 1-10^{-5}\right\}$.

\section{Model 2}

In order to apply the spectral methods to more general cases described above, we conduct 
Table 2: The queue length probability of Model 1

\begin{tabular}{ccccc}
\hline$n$ & $p_{n}$ & $F(n)$ & $q_{n}$ & $G(n)$ \\
\hline 0 & $1.1783495 \mathrm{E}-2$ & .01178349 & $5.0000000 \mathrm{E}-1$ & .50000000 \\
\hline 1 & $7.2293525 \mathrm{E}-3$ & .01901284 & $5.9763759 \mathrm{E}-3$ & .50597637 \\
\hline 2 & $7.0827611 \mathrm{E}-3$ & .02609560 & $3.5795161 \mathrm{E}-3$ & .50955589 \\
\hline 9 & $7.9749300 \mathrm{E}-3$ & .07882400 & $3.9413443 \mathrm{E}-3$ & .53569221 \\
\hline 10 & $4.5950561 \mathrm{E}-3$ & .08341906 & $4.0386703 \mathrm{E}-3$ & .53973088 \\
\hline 99 & $5.9452968 \mathrm{E}-3$ & .49747881 & $2.9429760 \mathrm{E}-3$ & .74642516 \\
\hline 100 & $2.7978123 \mathrm{E}-3$ & .50027662 & $3.007813 \mathrm{E}-3$ & .74943297 \\
\hline 1000 & $6.6051661 \mathrm{E}-6$ & .99906249 & $3.314196 \mathrm{E}-6$ & .99952959 \\
\hline 2000 & $5.9278497 \mathrm{E}-9$ & .99999976 & $2.9743465 \mathrm{E}-9$ & .99999988 \\
\hline
\end{tabular}

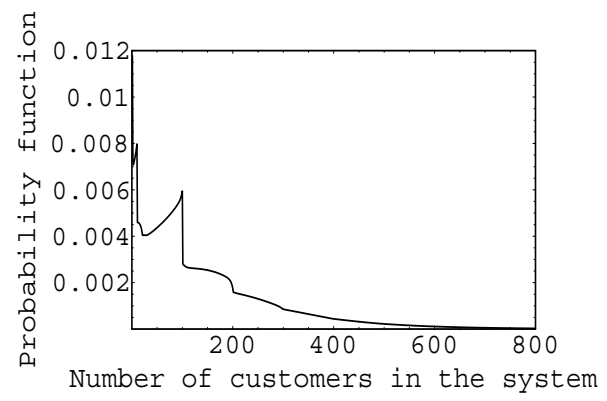

Figure 2: The probability function $p_{n}$

the following experiments. We fix $M=10$ and the arrival rate matrices $D_{k}$ given by

$$
\left\{\begin{array}{lll}
d_{i, i}^{\left(2^{i-1}\right)}=1 & (i=1,2, \ldots, 10) \\
d_{i, j}^{(k)} & =0 & \text { (otherwise) }
\end{array}\right.
$$

The off-diagonal elements of the matrix $D_{0}$ are randomly obtained as $P\left\{d_{i, j}^{(0)}=0\right\}=1 / 3$, $P\left\{d_{i, j}^{(0)}=0.1\right\}=1 / 3$ and $P\left\{d_{i, j}^{(0)}=1\right\}=1 / 3$. For $\rho=0.5$ we adopt a deterministic service time with $\mu=\lambda / 0.5$, where $\lambda$ is the arrival rate corresponding to a randomly constructed BMAP.

In randomly selected irreducible 100 cases, setting $K=1$ we success to obtain the stationary probability distributions in 76 cases but we fail in 24 cases. Then setting $K=2$ for the 12 cases in the remaining 24, the stationary probabilities are obtained. Table 4 shows the numbers of the cases that for $s=1, \ldots, K-1$ the stationary probability cannot be obtained and that for $s=K$ it can be obtained. The last remaining case requires $K=10$.

Table 3: Calculation time and $n^{*}$

\begin{tabular}{cccccccc}
\hline & $M$ & $a$ & $b$ & $\rho$ & $T_{1}$ (sec.) & $T_{2}$ (sec.) & $n^{*}$ \\
\hline The first case & 10 & 2 & 10 & 0.95 & 4.9 & 3523 & 18241 \\
\hline The second case & 31 & 1.1 & 2 & 0.5 & 134.4 & 38760 & 4037 \\
\hline
\end{tabular}

Table 4: The number of cases of the first success at $K$

\begin{tabular}{cccccccc}
\hline$K$ & 1 & 2 & 3 & 4 & 5 & $\cdots$ & 10 \\
\hline success/total & $76 / 100$ & $12 / 24$ & $7 / 12$ & $2 / 5$ & $2 / 3$ & $\cdots$ & $1 / 1$ \\
\hline
\end{tabular}




\section{Appendix}

In this appendix we discuss the spectral method for calculations of the stationary probability $\boldsymbol{p}_{n}$ and $\boldsymbol{q}_{n}$ using the Fourier inversion transform of the vector generating functions $\boldsymbol{p}(z)$ in (4.1) and $\boldsymbol{q}(z)$ in (4.2), respectively.

First we consider $\boldsymbol{p}_{n}$. Let $L$ be a sufficiently large integer such that the tail probability $\sum_{l=L}^{\infty} \boldsymbol{p}_{l} \boldsymbol{e}$ is negligible and define

$$
\begin{aligned}
\widetilde{\boldsymbol{p}}_{n} & =\sum_{l=0}^{\infty} \boldsymbol{p}_{l L+n} \quad(n=0, \ldots, L-1), \\
\widetilde{\boldsymbol{p}}(z) & =\sum_{k=0}^{L-1} \widetilde{\boldsymbol{p}}_{k} z^{k}
\end{aligned}
$$

Let $\omega=e^{\boldsymbol{i}_{2 \pi / L}}$ be the $L$ th root of the unity. Since for $n=l N+k \omega^{n}=\omega^{k}$ then $\widetilde{\boldsymbol{p}}\left(\omega^{k}\right)=$ $\boldsymbol{p}\left(\omega^{k}\right)$. From the Fourier inversion formula, we have for $n=0, \ldots, L-1$

$$
\boldsymbol{p}_{n} \approx \widetilde{\boldsymbol{p}}_{n}=\frac{1}{L} \sum_{k=0}^{L-1} \widetilde{\boldsymbol{p}}\left(\omega^{k}\right) \omega^{-k n}=\frac{1}{L} \sum_{k=0}^{L-1} \boldsymbol{p}\left(\omega^{k}\right) \omega^{-k n} .
$$

From Assumption 1 and the diagonal representation of $D(z) A(z)(z I-A(z))^{-1}$ we have Proposition 7.1 The stationary probability just after service completion epochs is given by

$$
\begin{aligned}
& \boldsymbol{p}_{n} \approx \frac{\lambda^{-1}(1-\rho) \boldsymbol{g}}{L} \\
& \sum_{k=0}^{L-1} V\left(\omega^{k}\right)\left[\begin{array}{ccc}
\frac{\alpha_{1}\left(\omega^{k}\right) h\left(\alpha_{1}\left(\omega^{k}\right)\right)}{\omega^{k}-h\left(\alpha_{1}\left(\omega^{k}\right)\right)} & & \\
& \ddots & \\
& & \frac{\alpha_{M}\left(\omega^{k}\right) h\left(\alpha_{M}\left(\omega^{k}\right)\right)}{\omega^{k}-h\left(\alpha_{M}\left(\omega^{k}\right)\right)}
\end{array}\right] U\left(\omega^{k}\right) \omega^{-k n} .
\end{aligned}
$$

In (7.1), for $k=0$ and $i=1$, we define as

$$
\frac{\alpha_{i}\left(\omega^{k}\right) h\left(\alpha_{i}\left(\omega^{k}\right)\right)}{\omega^{k}-h\left(\alpha_{i}\left(\omega^{k}\right)\right)}=\frac{\lambda}{1-\rho} .
$$

Similarly, we have

Proposition 7.2 The stationary probability at arbitrary time is given by

$$
\begin{aligned}
& \boldsymbol{q}_{n} \approx \frac{(1-\rho) \boldsymbol{g}}{L} \\
& \cdot \sum_{k=0}^{L-1} V\left(\omega^{k}\right)\left[\begin{array}{lll}
\frac{\left(\omega^{k}-1\right) h\left(\alpha_{1}\left(\omega^{k}\right)\right)}{\omega^{k}-h\left(\alpha_{1}\left(\omega^{k}\right)\right)} & & \\
& \ddots & \\
& & \frac{\left(\omega^{k}-1\right) h\left(\alpha_{M}\left(\omega^{k}\right)\right)}{\omega^{k}-h\left(\alpha_{M}\left(\omega^{k}\right)\right)}
\end{array}\right] U\left(\omega^{k}\right) \omega^{-k n} .
\end{aligned}
$$

In (7.2), for $k=0$ and $i=1$, we define as

$$
\frac{\left(\omega^{k}-1\right) h\left(\alpha_{i}\left(\omega^{k}\right)\right)}{\omega^{k}-h\left(\alpha_{i}\left(\omega^{k}\right)\right)}=\frac{1}{1-\rho} .
$$


It should be noted that the Fourier inversion transforms of the vector generating functions $\boldsymbol{p}(z)$ and $\boldsymbol{q}(z)$ are effectively calculated using the fast Fourier transform (FFT) in [23] for $L=2^{N}$, where $N$ is integer.

Remaining our problem is how to calculate $\alpha_{i}\left(\omega^{k}\right)(i=1, \ldots, M$ and $k=0, \ldots, L-1)$ and corresponding matrixes $U\left(\omega^{k}\right)$ and $V\left(\omega^{k}\right)$ at $(k=0, \ldots, L-1)$. The characteristic equation $\alpha I-D\left(\omega^{k}\right)$ of the matrix $D\left(\omega^{k}\right)$ is a polynomial of $\alpha$ with degree $M$. W apply the conventional D-K method in (5.1) to derive eigenvalues $\alpha_{i}\left(\omega^{k}\right)$. Setting the initial values of iterations as $\alpha_{i}\left(\omega^{k-1}\right)$, we calculate $\alpha_{i}\left(\omega^{k}\right)$ as

$$
\alpha_{i}^{(\nu+1)}=\alpha_{i}^{(\nu)}-\frac{\operatorname{det}\left(\alpha_{i}^{(\nu)} I-D\left(\omega^{k}\right)\right)}{\prod_{j=1, j \neq i}^{M}\left(\alpha_{i}^{(\nu)}-\alpha_{j}^{(\nu)}\right)} .
$$

This algorithm is efficient since the eigenvalue $\alpha_{i}\left(\omega^{k}\right)$ is closed to $\alpha_{i}\left(\omega^{k-1}\right)$ for a large $L$. After $\alpha_{i}\left(\omega^{k}\right)$ are calculated matrices $U\left(\omega^{k}\right)$ and $V\left(\omega^{k}\right)$ are easily calculated because $\operatorname{Rank}\left(\alpha_{i}\left(\omega^{k}\right) I-D\left(\omega^{k}\right)\right)=M-1$.

\section{References}

[1] O. Aberth: Iteration methods for finding all zeros of a polynomial simultaneously. Mathematics of Computation, 27 (1973) 339-344.

[2] D. Bini and B. Meini: On the solution of nonlinear matrix equation arising in queueing problems. SIAM Journal on Matrix Analysis and Applications, 17 (1996) 906-926.

[3] D. Bini and B. Meini: On cyclic reduction applied to a class of Toeplitz-like matrices arising in queueing problems, In W.J. Stewart (ed.): Computations with Markov Chains (Kluwer Academic Publishers, 1996) 203-217.

[4] H.R. Gail, S.L. Hantler and B.A. Taylor: Solutions of the basic matrix equation for M/G/1 and G/M/1 type Markov chains. Stochastic Models, 10 (1994) 1-43.

[5] H.R. Gail, S.L. Hantler and B.A. Taylor: Spectral analysis of M/G/1 and G/M/1 type Markov chains. Advances in Applied Probability, 28 (1996) 114-165.

[6] I. Kerner: Ein Gesamtschrittverfahren zur Berechnung der Nullstellen von Polynomen. Numerical Mathematics, 8 (1966) 290-294.

[7] D.M. Lucantoni: New results on the single server queue with a batch Markovian arrival process. Stochastic Models, 7 (1991) 1-46.

[8] D.M. Lucantoni and V. Ramaswami: Efficient algorithm for solving the non-linear matrix equations arising in phase type queues. Stochastic Models, 1 (1985) 29-51.

[9] B. Meini: An improved FFT-based version of Ramaswami's formula. Stochastic Models, 13 (1997) 223-238.

[10] M.F. Neuts: A versatile Markovian point process. Journal of Applied Probability, 16 (1979) 764-779.

[11] M.F. Neuts: Structured Stochastic Matrices of $M / G / 1$ Type and their Applications (Marcel Dekker Inc., New York), 1989.

[12] S. Nishimura: A spectral analysis for a MAP/D/N queue. In Latouche, G, Taylor, P. (eds.): Advances in Algorithmic Methods for Stochastic Models, (Notable Publications: 2000), 279-294.

[13] S. Nishimura: Spectral methods for a tree structure MAP. In G. Latouche, P. Taylor, (eds.), Matrix-Analytic Methods (World Scientific, 2002), 291-310. 
[14] S. Nishimura: A MAP/G/1 queue with a birth and death underlying process. Stochastic Models, 19 (2003) 425-447.

[15] S. Nishimura: A Spectral Method for a Nonpreemptive Priority BMAP/G/1 Queue. Stochastic Models, 21 (2005) 579-597.

[16] S. Nishimura and H. Sato: Eigenvalue expression for a batch Markovian arrival process. Journal of the Operations Research Society of Japan, 40 (1997) 122-132.

[17] S. Nishimura and M. Shinno: Modeling network traffic using a nearly completely decomposable TSMAP. Journal of the Operations Research Society of Japan, 47 (2004) 129-144.

[18] S. Nishimura, M. Shinno and T. Kanehori: Modeling IP traffic using a BMAP with short-term and long-term dynamics. Proceedings of ITC19, (2005) 929-938.

[19] V. Ramaswami: A stable recursion for the steady state vector in Markov chains of M/G/1 type. Stochastic Models, 4 (1988) 183-188.

[20] V. Ramaswami: Nonlinear matrix equations in applied probability-solution techniques and open problem. SIAM Review, 30 (1988) 256-263.

[21] S. Robert and J. Le Boudec: New models for pseudo self-similar traffic. Performance Evaluation, 30 (1997) 57-68.

[22] B. T. Smith: Error bounds for zeros of a polynomial based upon Gerschgorin's theorem. Journal of the ACM, 17 (1970) 661-674.

[23] H. Tijms: A First Course in Stochastic Models (Wiley, New York, 2003).

[24] J. Wilkinson:: The Algebraic Eigenvalue Problem (Clarendon Press, 1965).

[25] T. Yamamoto, S. Kanno and L. Atanassova: Validated computation of polynomial zeros by the Durand-Kerner method. In J. Herzberger, (ed.), Topics in Validated Computations, (Elesvier Science, 1994), 27-53.

Shoichi Nishimura,

Faculty of Science, Tokyo University of Science,

1-3 Kagurazaka, Shinjuku-ku,

Tokyo 162-8601, Japan.

E-mail: nishimur@rs.kagu.tus.ac.jp 